\title{
Gossypiboma- A Rare Cause of Gastric Outlet Obstruction
}

\author{
Parveen Malhotra*, Vani Malhotra, Naveen Malhotra, Ajay Chugh, Abhishek Chaturvedi, Parul Chandrika and \\ Ishita Singh \\ Department of Medical Gastroenterology, PGIMS, India
}

Submission: October 04, 2015; Published: November 10, 2015

*Corresponding author: Parveen Malhotra, Head and Department of Medical Gastroenterology, Obstetrics \& Gynecology and Anesthesiology, PGIMS, Rohtak 124001, Haryana, India, Tel: 9215372663; Email: drparveenmalhotra@yahoo.com

\begin{abstract}
Introduction: Gossypiboma is a surgical instrument or sponge left inside patient's body post operatively. It has varied manifestations from being asymptomatic to severe complications like vomiting, pain abdomen, obstruction, perforation, peritonitis and even death. Rarely it can have transmural migration and leads to bowel or visceral perforation, obstruction or fistula formation which usually occurs in stomach, ileum or colon. Very few cases have been reported in literature till now. We report the largest transmural migrated gossypiboma to date which was a surgical mop measuring $26 \times 23 \mathrm{~cm}$ and was successfully removed endoscopically.

Our Case: A fifty eight year-old man who was symptomatic for gallstones underwent open cholecystectomy six months back at some private hospital. He was asymptomatic for one month post-operatively but later on developed pain abdomen, dyspepsia, anorexia and intermittent vomiting. On investigating, barium swallow and abdominal ultrasonogram revealed a filling defect at antrum of stomach and duodenum which gave suspicion of malignancy. On upper gastrointestinal endoscopy, a surgical mop that had totally migrated into the stomach and duodenum was seen. The surgical mop was successfully removed by endoscopy and patient recovered completely after the procedure.
\end{abstract}

Conclusion: Gossypiboma should not be missed in any postoperative patient with unexplained pain abdomen and vomiting. Endoscopy is both diagnostic as well as therapeutic modality but surgery becomes definitive treatment in endoscopically failed cases or when incomplete migration of gossypiboma occurs into the stomach.

Keywords: Migratory gossypiboma; Pain abdomen; Vomiting; Endoscopy

\section{Introduction}

Gossypiboma is the term used to describe a retained non-absorbable surgical material that is composed of cotton matrix which leads to serious surgical complications for both patient and surgeon [1]. Clinical symptoms related to intraabdominal gossypiboma may vary from mild abdominal pain to major complications including bowel or visceral perforation, obstruction, fistula formation or sepsis [2]. Despite its rarity, transmural migration of gossypiboma is one of the possible causes of these gastrointestinal complications. Transmural migration of an intra-abdominal gossypiboma has been reported to occur in stomach, ileum, colon, bladder, vagina and diaphragm [3]. As per literature, this is the sixth reported case of transgastric migration of a gossypiboma but all of them were surgical sponges of smaller size and three of them were removed endoscopically $[3,4]$.

\section{Case Report}

A fifty eight year-old man underwent open cholecystectomy for cholelithiasis, six months back but four weeks post- operatively he developed pain abdomen and persistent vomiting. Persistent abdominal pain was mainly in the epigastrium and right hypochondrium. He was symptomatically treated by various private practitioners but in vain. In between he had a bout of fever which was associated with rigor and chills and remained for one week. He became afebrile after five days of oral antibiotics treatment but pain abdomen and vomiting persisted. Patient had features of complete gastric outlet obstruction for last three weeks, before he reported to our department. He was not able to accept anything orally and was on intravenous fluids. On investigating, he had mild anemia, but liver and renal function tests were normal. The barium swallow (Figure 1) and abdominal ultrasonogram revealed a filling defect at antrum of stomach and duodenum which was suggestive of gastric carcinoma. Thus endoscopy was done for proper diagnosis, after obtaining written informed consent from the patient including surgical risks. Endoscopy revealed a large surgical sponge which totally migrated and was found partially in pyloric antrum and rest half was stuck in duodenum (Figure 2). The surgical sponge was loosened with normal saline and then with saw-tooth forceps was pulled into the stomach (Figure 3). The sponge was 
then removed with gentle round motions from the mouth. The prolonged sticking of surgical sponge lead to huge dilatation of pyloric antrum (Figure 4). The removed surgical sponge was 26 $\mathrm{cm} \times 23 \mathrm{~cm}$ and is of the largest size which has been successfully removed endoscopically, till date (Figure 5). The procedure was completed without any complications .The next day; all laboratory parameters, check endoscopy and abdominal ultrasonogram were found to be normal. The patient was started on per- oral feeds which he accepted well, hence was discharged under hemodynamically normal condition after two days of observation.

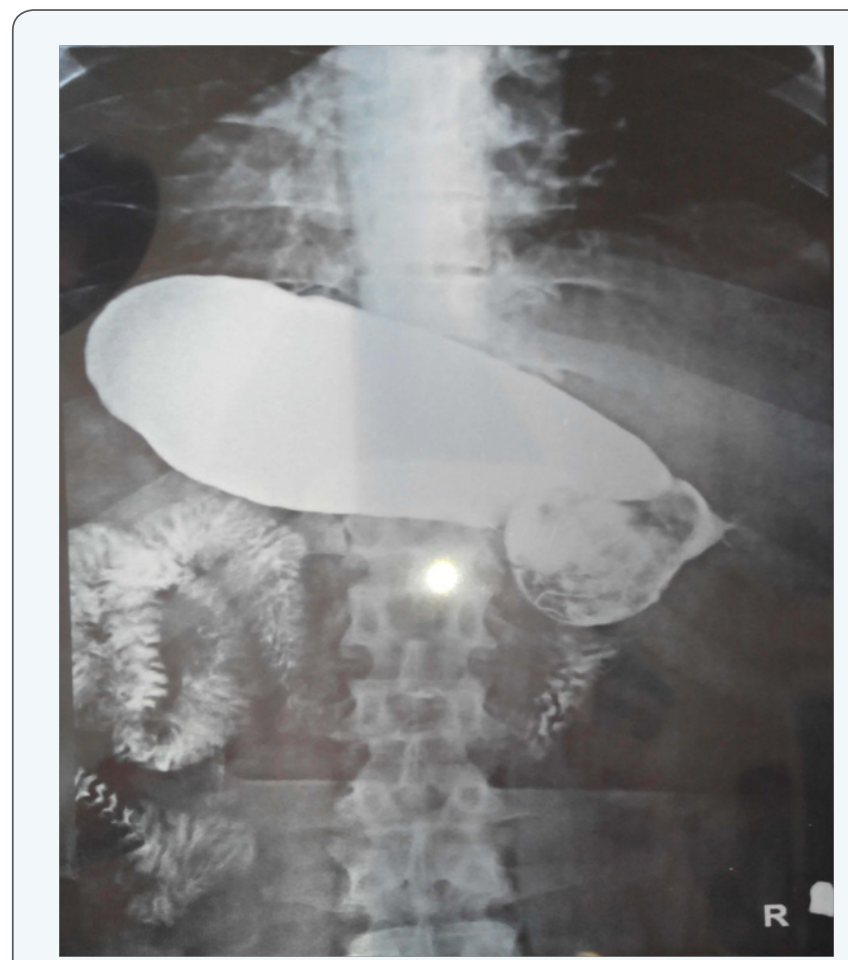

Figure 1: Barium swallow revealing mass like lesion at Antro-duodenal area.

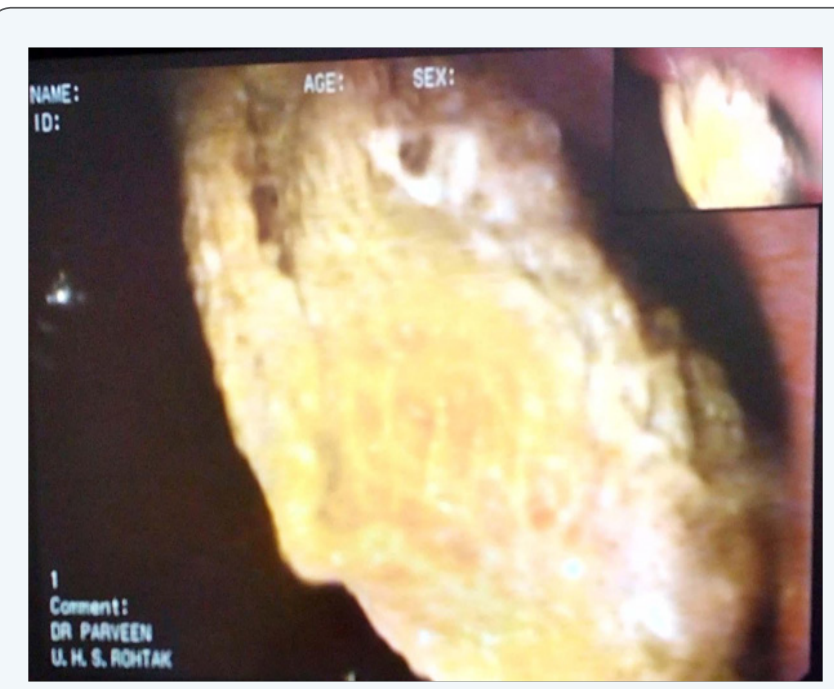

Figure 2: Large Gossypiboma which migrated into stomach.

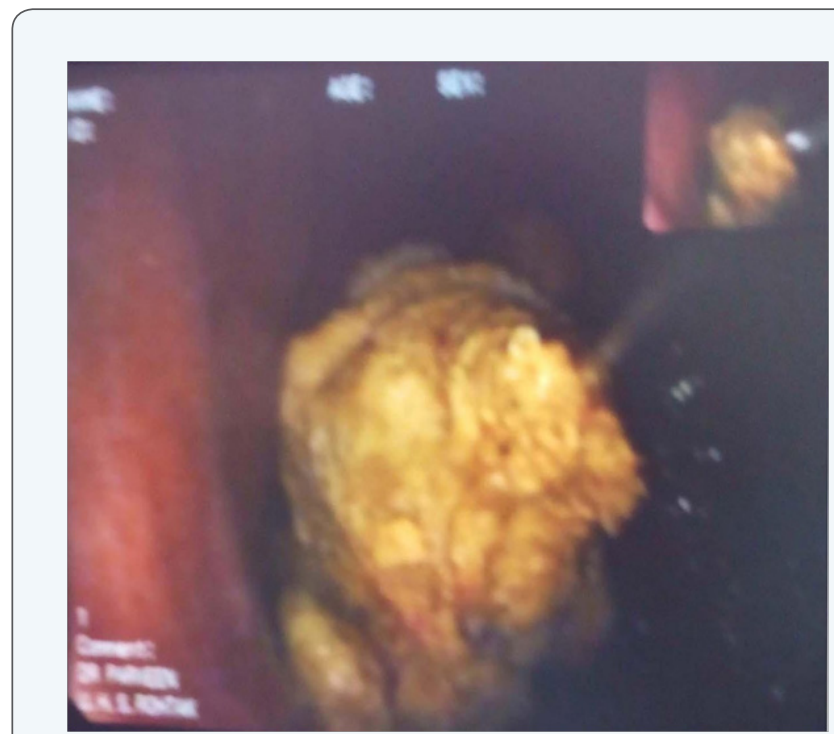

Figure 3: Gossypiboma being removed with help of Snare.

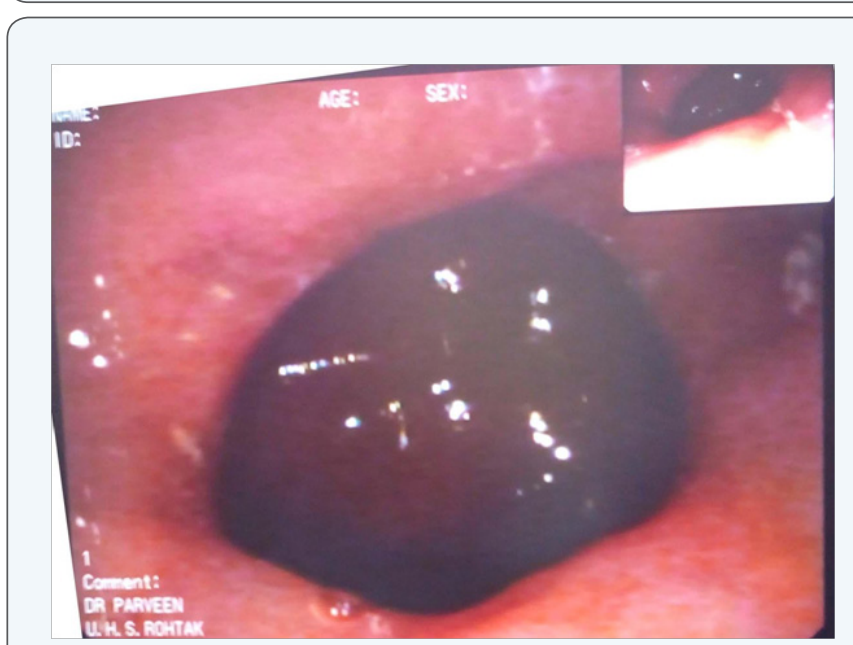

Figure 4: Dilated Pyloric Antrum after removal stucked Gossypiboma.

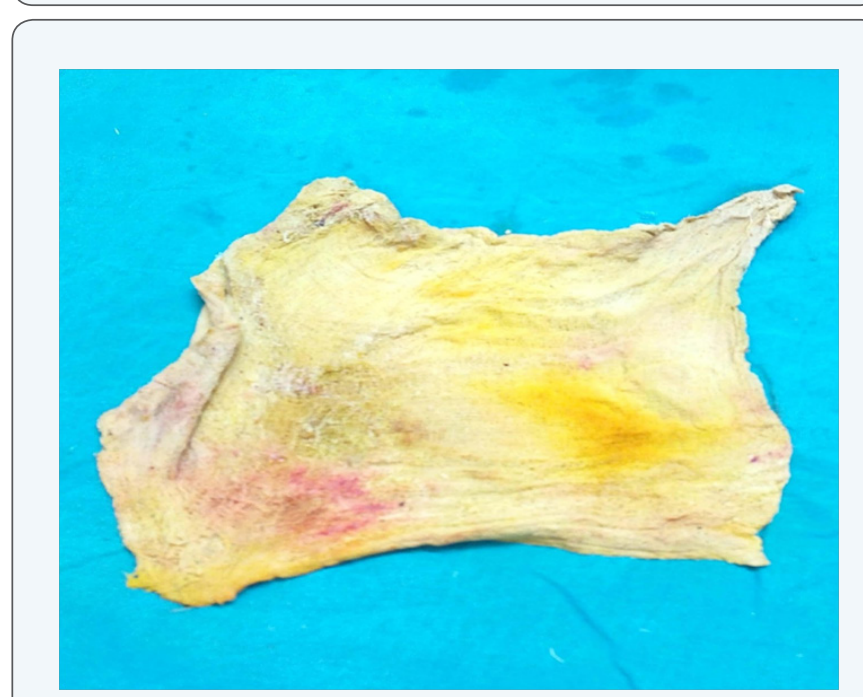

Figure 5: The removed surgical sponge of size $26 \mathrm{~cm} \times 23 \mathrm{~cm}$. 


\section{Discussion}

Gossypiboma following an intra-abdominal surgery is potentially dangerous and it is encountered more commonly than is reported [5]. Gossypiboma leads to two types of foreign body reactions; aseptic fibrinous response and an exudative reaction [6,7]. The aseptic fibrinous response can be asymptomatic or gives rise to painless abdominal mass, whereas exudative reactions can lead to severe manifestations like intestinal perforation, obstruction, fistula formation or sepsis [8]. Retained sponge migrates rarely and is bodily response to extrude the foreign material by developing a fistula externally or into a hollow viscus. Transmural migration occurs as a result of inflammation in the intestinal wall which ultimately leads to necrosis [9]. Later on, necrotic area closes after complete migration of the surgical sponge. The small intestine is the most affected site due to its thin wall and large outer surface. Compared with the intestines, the stomach is an unusual site for transmural migration due to its higher localization and thick wall [9]. The same thing occurred in our case as patient developed gradually progressive features of obstruction and had an episode of fever associated with rigor and chills which may have been due to mild peritonitis, at the time of migration of surgical sponge into gastrointestinal tract. The patient developed features of complete gastro-duodenal outlet obstruction, due to complete sticking of sponge at pyloric antro-duodenal level. Until now, this condition has been previously reported only in five cases $[3,4,6,9]$. Interestingly, all of them occurred after acute open cholecystectomy operations, as occurred in our case. Hence, we emphasize that acute cholecystectomy is a major factor that leads to this kind of complication.

Imaging procedures such as plain X-ray, USG, CT and/or magnetic resonance (MR) may usually be helpful for diagnosis. In addition, imaging of a hyperechogenic mass with hypoechoic rim on USG or a rounded mass with a dense central part and enhancing wall on CT are the basic signs of gossypiboma $[5,10]$. It is misdiagnosed as intra-abdominal hematoma, abscess or neoplasm which leads to unnecessary radical surgical interventions.

Gossypiboma should be removed as soon as possible to avoid further surgical complications and legal problems [1]. Although open surgery is the most common approach in the treatment of gossypiboma, removal can be easily performed by minimally invasive techniques such as endoscopy or laparoscopy $[1,4,6]$. Although successful removals of surgical sponges by endoscopy have been reported before, the feasibility of endoscopy in removal of such a large surgical sponge compress was challenging. To our knowledge, herein we report the first case of the largest gossypiboma published till date successfully treated endoscopically. Hence, we emphasize that endoscopy may be a good option in the removal of such a large sponge located in the stomach and duodenum. However, surgery should be considered when fixed reaction and/or partial migration have occurred.

Patients undergoing emergency surgery, those with high body mass index, lengthy operations, inexperienced staff or unexpected change in surgical procedure are major risk factors for retained surgical materials $[1,8]$. Simple precautions like educating the staff, tagging the sponges with markers or preoperative multiple counts of sponges and materials should reduce the incidence of gossypiboma [8]. In addition, new technologies like electronic tagging of sponges may be helpful in decreasing the incidence [11].

\section{Conclusion}

Transmural migration of gossypiboma should be considered in the differential diagnosis of any postoperative patient with unexplained pain abdomen and vomiting. Endoscopy is both diagnostic as well as therapeutic modality but surgery may be considered in endoscopically failed/ refractory cases or in cases of incomplete migration of gossypiboma.

\section{References}

1. Sozutek A, Karabuga T, Bozdag AD, Derici H (2010) Asymptomatic gossypiboma mimicking a liver mass. Turk J Surg 26(4): 225-228.

2. Gawande AA, Studdert DM, Orav EJ, Brennan TA, Zimmer MJ (2003) Risk factors for retained instruments and sponges after surgery. N Eng J Med 348(3): 229-235.

3. Erdil A, Kilciler G, Ates Y, Tuzun A, Gulsen M, et al. (2008) Transgastric migration of retained intraabdominal surgical sponge: gossypiboma in the bulbus. Inter Med 47(7): 613-615.

4. Mentes BB, Yilmaz E, Sen M, Kayhan B, Gorgul A, et al. (1997) Transgastric migration of a surgical sponge. J Clin Gastroenterol 24(1): 55-57.

5. Cheng TC, Chou AS, Jeng CM, Chang PY, Lee CC (2007) Computed tomography findings of gossypiboma. J Chin Med Assoc 70(12): 565569.

6. Erbay G, Koc Z, Caliskan K, Araz F, Ulusan S (2012) Imaging and clinical findings of a gossypiboma migrated into the stomach. Turk J Gastroenterol 23(1): 54-57.

7. Düx M, Ganten M, Lubienski A, Grenacher L (2002) Retained surgical sponge with migration into the duodenum and persistent duodenal fistula. Eur Radiol 12(Suppl 3): 74-77.

8. Patil KK, Patil SK, Gorad KP, Panchal AH, Arora SS, et al. (2010) Intraluminal migration of surgical sponge: gossypiboma. Saudi J Gastroenterol 16(3): 221-222.

9. Sarda AK, Pandey D, Neogi S, Dhir U (2007) Postoperative complications due to a retained surgical sponge. Singapore Med J 48(6): e160-164.

10. Kopka L, Fischer U, Gross AJ, Funke M, Oestmann JW, et al. (1996) CT of retained surgical sponges (textilomas): pitfalls in detection and evaluation. J Comput Assist Tomogr 20(6): 919-923.

11. Fabian CE (2005) Electronic tagging of surgical sponges to prevent their accidental retention. Surgery 137(3): 298-301. 